\title{
Determinação experimental da viscosidade e condutividade térmica de óleos vegetais
}

\author{
Experimental measurements of viscosity and thermal conductivity of vegetable oils \\ Josiane BROCK ${ }^{1}$, Maria Rita NOGUEIRA ${ }^{1}$, Cláudio ZAKRZEVSKI ${ }^{1}$, Fernanda de Castilhos CORAZZA ${ }^{1}$, \\ Marcos Lúcio CORAZZA ${ }^{1}$, José Vladimir de OLIVEIRA ${ }^{1 *}$
}

\section{Resumo}

O presente trabalho tem por objetivo reportar valores experimentais de condutividade térmica e viscosidade dinâmica dos óleos vegetais refinados de soja, milho, girassol, algodão, canola, oliva e de farelo de arroz. As medidas de condutividade térmica foram realizadas em célula acoplada a um banho termostático no intervalo de temperatura de 20 a $70{ }^{\circ} \mathrm{C}$, utilizando uma sonda de fio quente. Os resultados obtidos demonstram que para todos os óleos vegetais investigados a condutividade térmica possui fraca dependência com a temperatura, apresentando ligeiro decréscimo com o aumento desta variável. O método gravimétrico foi empregado para a medida da densidade dos óleos vegetais estudados à temperatura ambiente, não tendo sido verificada diferença significativa entre os valores encontrados. Para as medidas de viscosidade dos óleos vegetais foi utilizado um viscosímetro do tipo Brookfield, acoplado a um banho termostatizado com controle de temperatura. A partir dos resultados obtidos verificou-se que a viscosidade decresce acentuadamente com o aumento da temperatura para todos os óleos vegetais.

Palavras-chave: óleo vegetal; densidade; viscosidade; condutividade térmica.

\begin{abstract}
This work reports experimental data of thermal conductivity and dynamic viscosity of the following refined vegetable oils: rice, soybean, corn oil, sunflower, cottonseed, and olive oil. Measurements of thermal properties were carried out in a cell coupled to a thermostatic bath in the temperature range of $20-70^{\circ} \mathrm{C}$, using a single-needle stainless steel sensor. It was experimentally observed that the thermal conductivity decreased slightly with increasing temperature for all samples investigated. The gravimetric method was employed for density data acquisition, and revealed no significant difference among the values obtained. The Brookfield apparatus was employed in measuring the dynamic viscosity and it was verified that a raise in temperature led to a sharp decrease for this property for all samples investigated.
\end{abstract}

Keywords: vegetable oil; density; viscosity; thermal conductivity.

\section{Introdução}

Tem se verificado atualmente um aumento acentuado na demanda de mercado em relação a óleos vegetais das mais diversas fontes naturais, destacando-se as aplicações em derivados alimentícios que cobrem desde a formulação de produtos, em que a viscosidade para alimentos líquidos é parâmetro fundamental para caracterização e avaliação de textura destes, até a transformação e obtenção de ésteres a partir de triacilglicerídeos, ou ainda o emprego dos óleos vegetais diretamente na formulação de combustíveis minerais (CONCEIÇÃO et al., 2005). Qualquer que seja a forma de obtenção ou emprego dos óleos vegetais, o conhecimento de propriedades termofísicas, tais como densidade, viscosidade, condutividade e difusividade térmica é de fundamental importância para a consecução das etapas de projeto de equipamentos e de processos ou mesmo para especificação do produto.

Até o presente, poucos estudos referentes ao comportamento reológico de óleos vegetais têm sido reportados na literatura (SANTOS et al., 2005; ENCINAR et al., 2002). Alguns trabalhos apresentados, no entanto, referem-se a tais medidas para tria- cilglicerídeos puros e misturas binárias de triacilglicerídeos de cadeias curtas (EITMAN; GOODRUM, 1993; RABELO et al., 2000). No entanto, informações sobre propriedades térmicas, especificamente, condutividade e difusividade térmica de óleos vegetais são escassas.

$\mathrm{Na}$ indústria de alimentos, contudo, o conhecimento de tais propriedades se faz necessário para o projeto e desenvolvimento de cálculos, de equipamentos e processos que envolvam transferência de calor, podendo-se citar o exemplo de projetos para equipamentos voltados à refrigeração, tratamento térmico e armazenamento de alimentos.

Geralmente, nas determinações experimentais das propriedades térmicas de alimentos, a maior dificuldade é atribuída à grande dependência destas em relação à temperatura e composição. Ainda, a maioria dos estudos envolvendo o desenvolvimento de modelos matemáticos e medidas experimentais de propriedades térmicas de alimentos é realizada utilizando sistemas modelo, e os resultados são aplicados para alimentos

Recebido para publicação em 2/3/2007

Aceito para publicação em 5/8/2007 (002343)

${ }^{1}$ Departamento de Engenharia de Alimentos, Universidade Regional Integrada - URI, Campus de Erechim, Av. Sete de Setembro, 1621, CEP 99700-000, Erechim - RS, Brasil, E-mail:vladimir@uricer.edu.br

${ }^{*}$ A quem a correspondência deve ser enviada 
de composição similar (RESENDE; SILVEIRA, 2002). Desta forma, dados de propriedades como condutividade térmica, viscosidade, difusividade térmica e densidade apresentam papel preponderante no contexto de processamento de óleos vegetais.

Neste contexto, o presente trabalho tem por objetivo apresentar resultados experimentais de medidas de viscosidade de diversos óleos vegetais comerciais, bem como a caracterização reológica destes óleos e medidas de propriedades térmicas condutividade e difusividade térmica. Para tal, foram avaliadas amostras comerciais dos óleos refinados de soja, milho, algodão, canola, oliva, girassol e de farelo de arroz.

\section{Material e métodos}

\subsection{Materiais}

Todos os óleos vegetais utilizados no presente trabalho (milho - marca Salada, soja - marca Soya, algodão - marca Salada, oliva - marca Salada, girassol - marca Salada, canola - marca Salada e de farelo de arroz - marca Carreteiro) foram adquiridos no mercado local e usados sem nenhum tratamento adicional.

\subsection{Procedimento experimental}

\section{Medidas de densidade}

As densidades foram medidas em temperatura ambiente $\left(25 \pm 1{ }^{\circ} \mathrm{C}\right)$ e para tal, as amostras foram pesadas em balões volumétricos de $10 \mathrm{~mL}$, previamente aferidos. Após a pesagem a densidade foi determinada utilizando a relação entre massa medida (em balança analítica com quatro casas de precisão marca Gibertini modelo EC154) e o volume do balão utilizado (previamente aferido com água bidestilada à temperatura de $25^{\circ} \mathrm{C}$ ). O procedimento de medida para todas as amostras foi realizado em triplicata, tendo sido obtido então um valor de densidade média medida e seu respectivo desvio padrão $(\sigma)$.

\section{Medidas de viscosidade}

Para a determinação da viscosidade dos diferentes tipos de óleos vegetais refinados foi utilizado um viscosímetro da marca Brookfield (Modelo LVDV-III+). O instrumento é equipado com cilindros de diâmetros diferentes (spindles), em que é utilizado o cilindro adequado conforme a viscosidade do fluido. Para os óleos utilizados neste trabalho foi utilizado um cilindro de diâmetro externo de $100 \mathrm{~mm}$ (Spindle de referência S-28).

O viscosímetro foi acoplado a um banho termostático, permitindo assim mensurar a viscosidade dos óleos no intervalo de 20 a $70^{\circ} \mathrm{C}$, com precisão na temperatura de $0,5^{\circ} \mathrm{C}$. Uma vez que o software do viscosímetro fornece, além dos valores de viscosidade, os dados de tensão de cisalhamento em função da taxa de cisalhamento, estes foram utilizados para a caracterização reológica das amostras.

Cabe ressaltar que no viscosímetro de Brookfield são efetuadas leituras de viscosidade automaticamente à cada temperatura, variando-se a velocidade de rotação do cilindro (torque) até o limite máximo estabelecido, e ao atingir o valor de topo desta variável, medidas são efetuadas com o decréscimo desta. Em outras palavras, os valores de viscosidade reportados neste trabalho referem-se de fato a valores médios, obtidos por triplicata de leitura do equipamento em cada valor de velocidade de rotação especificada. O desvio padrão médio global para todos os óleos vegetais investigados no presente trabalho foi de $\pm 0,5 \mathrm{mPa} . \mathrm{s}^{-1}$.

\section{Medidas de condutividade e difusividade térmica}

Para as medidas de condutividade e difusividade térmica dos óleos vegetais foi utilizado um analisador de propriedades térmicas (Decagon Inc., modelo KD2). Para tal, foi utilizada uma célula de vidro (capacidade $40 \mathrm{~mL}$ ) encamisada acoplada a um banho termostático para controle da temperatura. As leituras foram feitas em triplicata, no intervalo de temperatura de 20 a $70^{\circ} \mathrm{C}$.

$\mathrm{O}$ princípio de funcionamento da sonda KD2 baseia-se na metodologia de fio quente, em que os valores de $\mathrm{k}$ (condutividade térmica) e $\alpha$ (difusividade térmica) são obtidos através da solução da Equação de condução de calor em coordenadas cilíndricas em um meio homogêneo (Equação 1) (FONTANA et al., 2001):

$\frac{d T}{d t}=\alpha\left(\frac{\mathrm{d}^{2} \mathrm{~T}}{d r^{2}}+r^{-1} \frac{d T}{d r}\right)$

em que:

$\mathrm{T}=$ temperatura $\left({ }^{\circ} \mathrm{C}\right)$;

$\mathrm{t}=$ tempo $(\mathrm{s})$;

$\alpha=$ difusividade térmica $\left(\mathrm{m}^{2} \mathrm{~s}^{-1}\right)$; e

$\mathrm{r}=\operatorname{distância}(\mathrm{m})$.

A solução analítica da Equação 1 pode ser dada por Fontana et al. (2001) por meio da Equação 2:

$T-T_{0}=\left(\frac{q}{4 \pi k}\right) E_{i}\left(-\frac{r^{2}}{4 \alpha t}\right)$

em que:

$\mathrm{q}=$ quantidade de calor produzida por unidade de tempo (W);

$\mathrm{k}=$ condutividade térmica medida $\left(\mathrm{W} \mathrm{m}^{-1}{ }^{\circ} \mathrm{C}^{-1}\right)$; e

$\mathrm{E}_{\mathrm{i}}=$ função exponencial integral, dada pela Equação 3:

$-E i(-a)=\int_{a}^{\infty}\left(\frac{1}{u}\right) \exp (u-1) d u=-\gamma-\ln \left(\frac{r^{2}}{4 \alpha t}\right)+\frac{r^{2}}{4 \alpha t}-\left(\frac{r^{2}}{8 \alpha t}\right)^{2}+\ldots$

em que, $a=r^{2} / 4 \alpha t$ e $\gamma$ é uma constante $(0,5772 \ldots)$.

Para valores de $\mathrm{t}$ suficientemente grandes, os termos de ordem mais elevada podem ser ignorados. Assim, a partir das Equações 2 e 3 tem-se a Equação 4:

$$
T-T_{0} \cong \frac{q}{4 \pi k}\left[\ln (t)-\gamma-\ln \left(\frac{r^{2}}{4 \alpha}\right)\right]
$$

A Equação 4 representa a relação entre a condutividade térmica e o deslocamento ( $\left.\mathrm{T}-\mathrm{T}_{0}\right)$. Esse descolamento e $\ln (\mathrm{t})$ estão correlacionados linearmente pela inclinação $(m=q / 4 \pi k)$. Desta 
forma, a condutividade pode ser obtida a partir do coeficiente linear de (4), (Equação 5) em que:

$k \cong \frac{q}{4 \pi m}$

A difusividade térmica pode também ser obtida a partir da Equação 4, registrando-se a interseção da linha de regressão $\left(\mathrm{T}-\mathrm{T}_{0}\right.$ ) versus $\ln \mathrm{t}$, com $\mathrm{r}$ finito, de acordo com a Equação 6:

$\ln \left(t_{0}\right)=\left[\gamma+\ln \left(\frac{r^{2}}{4 \alpha}\right)\right]$

\section{Resultados e discussão}

Inicialmente, visando à validação da sonda KD2 e ao procedimento de medida, foram realizados inicialmente alguns testes, utilizando para fins de comparação valores de $\mathrm{k}$ e $\alpha$ para algumas substâncias disponíveis na literatura. Tais resultados estão apresentados na Tabela 1 e demonstram a boa concordância encontrada entre os valores obtidos neste trabalho e aqueles da literatura.

\subsection{Densidade dos diferentes óleos vegetais}

$\mathrm{Na}$ Tabela 2 são apresentados os valores de densidade e desvios padrão obtidos para os diferentes óleos vegetais investigados no presente trabalho. Pode ser observado, a partir desta tabela, que na condição de medida a densidade dos diferentes óleos vegetais refinados não apresenta diferença considerável entre as amostras.

\subsection{Medidas de viscosidade}

Nesta seção são apresentados os resultados das medidas de viscosidade, bem como das análises referentes ao comportamento reológico dos diferentes óleos vegetais. Cabe ressaltar, que informações desta natureza são relativamente escassas na literatura.

Para a avaliação do comportamento reológico dos óleos vegetais estudados foram avaliados diagramas de tensão de cisalhamento $\left(\mathrm{N} / \mathrm{m}^{2}\right)$ em função da taxa de cisalhamento $\left(\mathrm{s}^{-1}\right)$ para as temperaturas de 20 e $70{ }^{\circ} \mathrm{C}$, os quais foram obtidos a partir das medidas realizadas no viscosímetro. Na Figura 1 são apresentados os diagramas de tensão de cisalhamento em função da taxa de cisalhamento para o óleo de milho para as temperaturas de 20 e $70{ }^{\circ} \mathrm{C}$. Nesta análise foi aplicado um modelo linear (linha contínua) para caracterização do comportamento reológico (relação de Newton da viscosidade), não tendo sido observada ocorrência de histerese.

Na Figura 2 é apresentado o diagrama da viscosidade em função da taxa de cisalhamento para o óleo de milho na temperatura de $20^{\circ} \mathrm{C}$. A partir desta figura pode-se observar uma variação significativa nos valores de viscosidade para baixas taxas de cisalhamento aplicadas $\left(<5 \mathrm{~s}^{-1}\right)$, indicando um comportamento não Newtoniano do óleo, ou ainda, classificação como fluido de Bingham (2005). Para valores de taxa de cisalhamento superiores a $5 \mathrm{~s}^{-1}$ o comportamento verificado é do tipo fluido-Newtoniano, uma vez que a viscosidade é constante para qualquer valor de taxa de cisalhamento.

Destaca-se que o mesmo comportamento reológico observado para o óleo de milho, explicitado nas Figuras 1 e 2, foi verificado para os demais óleos vegetais investigados neste trabalho. De maneira geral, constatou-se que o comportamento reológico dos óleos vegetais pode ser classificado como Newtoniano, exceto para baixos valores de taxa de cisalhamento $\left(<5 \mathrm{~s}^{-1}\right)$, uma vez que na faixa de temperatura investigada no presente trabalho verificou-se uma variação linear (com coeficiente linear igual a zero) da tensão de cisalhamento em função da taxa de cisalhamento. Tal comportamento está de acordo com os resultados apresentados na literatura para alguns óleos vegetais (CONCEIÇÃO et al., 2005; SANTOS, 2005). Na Figura 3 são apresentados os resultados da caracterização reológica para o óleo de algodão, soja, girassol, canola, oliva e de farelo de arroz, num diagrama da tensão de cisalhamento em função da taxa de cisalhamento.

A Tabela 3 apresenta os valores de viscosidade medidos para os diferentes óleos vegetais no intervalo de temperatura de 20 a $70{ }^{\circ} \mathrm{C}$, obtidos a uma taxa de cisalhamento constante para todos os óleos de $40 \mathrm{~s}^{-1}$.

A Figura 4 proporciona visualização adequada dos valores de viscosidade dos óleos vegetais em função da temperatura, apresentados na Tabela 3.

Tabela 1. Comparação entre valores medidos e da literatura para condutividade térmica $(\mathrm{k})$, difusividade térmica $(\alpha)$ e capacidade calorífica $\left(c_{p}\right)$ - calibração da sonda KD2.

\begin{tabular}{|c|c|c|c|c|c|}
\hline \multicolumn{3}{|c|}{ Este trabalho } & \multicolumn{3}{|c|}{ Literatura } \\
\hline $\mathrm{T}\left({ }^{\circ} \mathrm{C}\right)$ & $\mathrm{k}\left(\mathrm{W} \cdot \mathrm{m}^{-1}{ }^{\circ} \mathrm{C}\right)$ & $\alpha\left(\mathrm{mm}^{2} / \mathrm{s}\right)$ & $\mathrm{T}\left({ }^{\circ} \mathrm{C}\right)$ & $\mathrm{k}\left(\mathrm{W} \cdot \mathrm{m}^{-1}{ }^{\circ} \mathrm{C}\right)$ & $\alpha\left(\mathrm{mm}^{2} / \mathrm{s}\right)$ \\
\hline \multicolumn{6}{|l|}{ Água } \\
\hline 18,0 & 0,590 & 0,15 & 17,6 & $0,61^{\mathrm{a}}$ & $0,143^{c}$ \\
\hline \multicolumn{6}{|c|}{ Óleo de mamona } \\
\hline 20,0 & 0,169 & 0,10 & 19,6 & $0,17^{\mathrm{a}}$ & - \\
\hline \multicolumn{6}{|l|}{ Glicerina } \\
\hline 20,0 & 0,284 & 0,10 & 20,0 & $0,286^{a}$ & 0,0955 \\
\hline \multicolumn{6}{|l|}{ Etilenoglicol } \\
\hline 27,1 & 0,240 & 0,09 & 27,0 & $0,252^{\mathrm{b}}$ & $0,0939^{b}$ \\
\hline 37,0 & 0,240 & 0,09 & 37,0 & $0,255^{\mathrm{b}}$ & $0,0939^{b}$ \\
\hline 47,1 & 0,240 & 0,09 & 47,0 & $0,258^{\mathrm{b}}$ & $0,0940^{\mathrm{b}}$ \\
\hline
\end{tabular}

a(FONTANA et al., 2001); '(INCROPERA; DE WITT, 1990); e c(WELTY; WICKS; WILSON, 1984). 
Tabela 2. Valores medidos de densidade dos diferentes óleos vegetais à temperatura ambiente $\left(25 \pm 1^{\circ} \mathrm{C}\right)$.

\begin{tabular}{lccccccc}
\hline & \multicolumn{7}{c}{ Óleo } \\
\cline { 2 - 8 } & Soja & Milho & Arroz & Girassol & Algodão & Oliva & Canola \\
\hline$\rho\left(\mathrm{g} / \mathrm{cm}^{3}\right)$ & 0,883 & 0,875 & 0,877 & 0,877 & 0,875 & 0,879 & 0,878 \\
$\pm \sigma$ & 0,004 & 0,003 & 0,002 & 0,004 & 0,002 & 0,003 & 0,002 \\
\hline
\end{tabular}

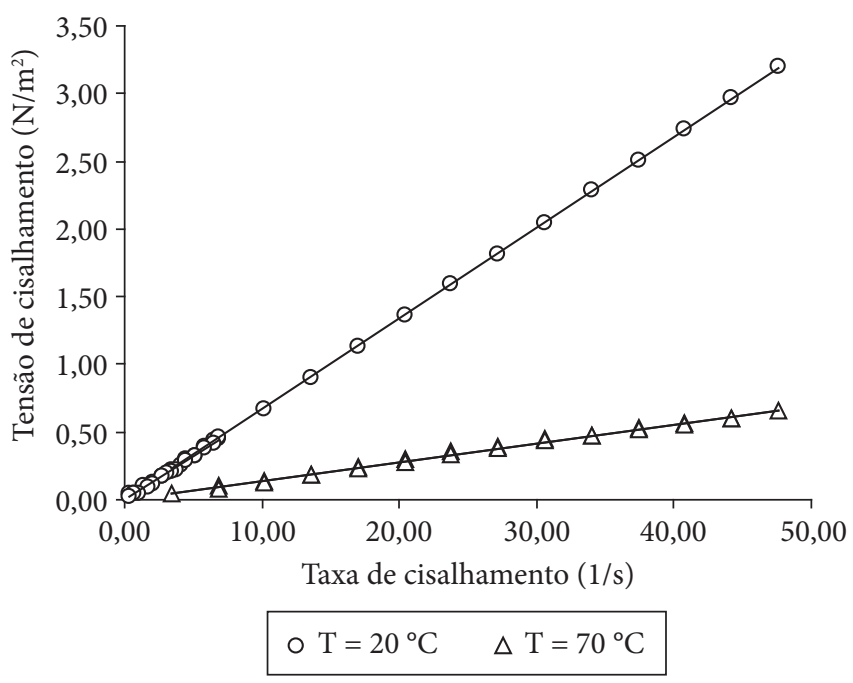

Figura 1. Comportamento reológico para o óleo de milho.

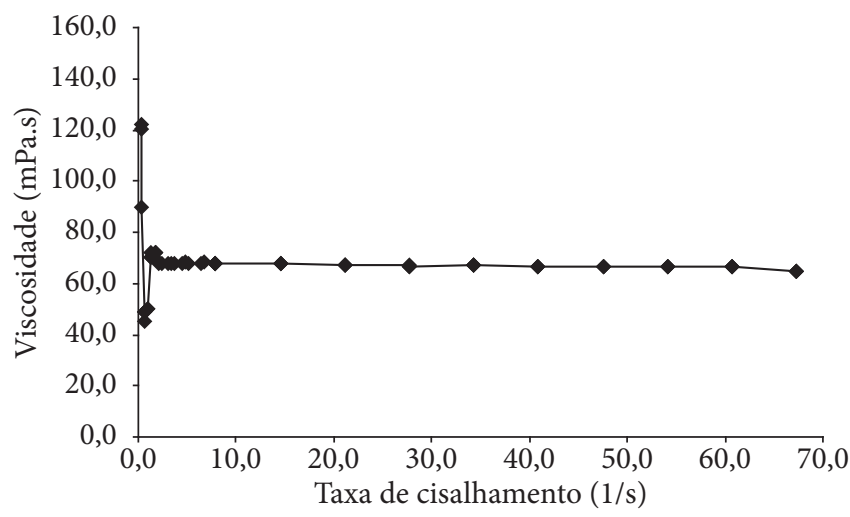

Figura 2. Diagrama de viscosidade em função da taxa de cisalhamento para o óleo de milho a $20^{\circ} \mathrm{C}$.

Pode-se observar, a partir da Figura 4, que há uma diminuição acentuada na viscosidade de todos os óleos vegetais investigados à medida que a temperatura é acrescida. Observase ainda que, para valores de baixa temperatura, ocorre uma diferença mais nítida entre os valores de viscosidade quando comparados às temperaturas mais elevadas.

Inicialmente, para as medidas de viscosidade do óleo de soja obtidas no presente trabalho, foi realizada uma modelagem de viscosidade em função da temperatura. Para tal, foram aplicados dois modelos, de Guzman (Equação 7) e de Vogel (Equação 8), os quais são tradicionalmente usados na literatura (REID; PRAUSNITZ; POLING, 1988):

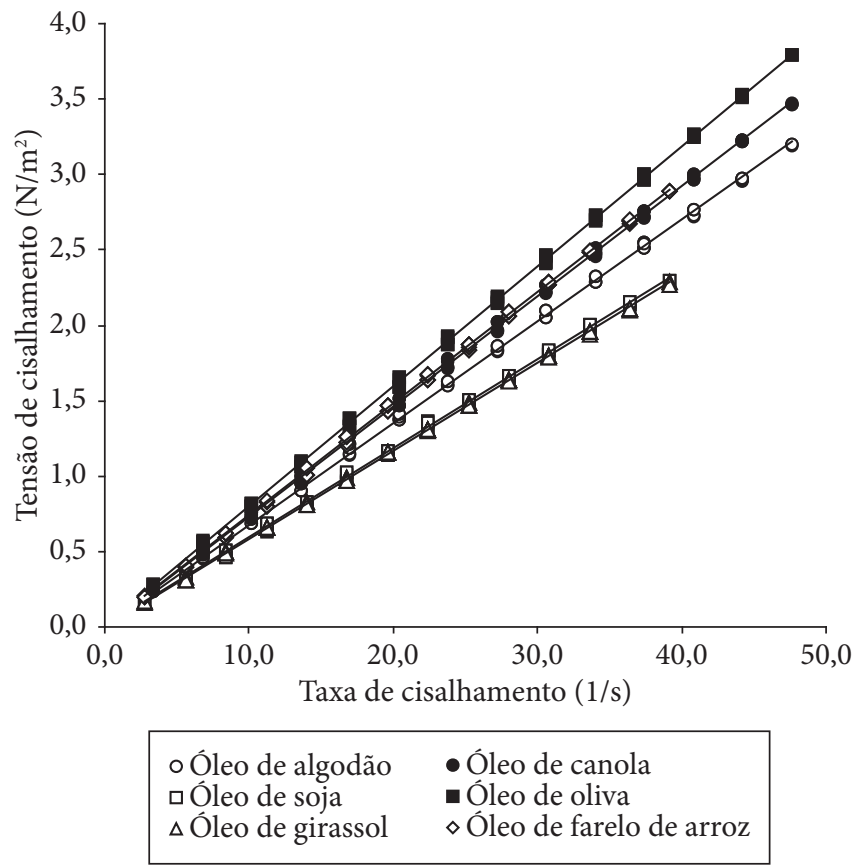

Figura 3. Comportamento reológico para os diversos óleos vegetais a $20^{\circ} \mathrm{C}$.

Tabela 3. Valores de viscosidade medidos para os diferentes óleos vegetais em função da temperatura.

\begin{tabular}{lccccccc}
\hline $\mathrm{T}\left({ }^{\circ} \mathrm{C}\right)$ & \multicolumn{7}{c}{ Viscosidade (mPa.s) } \\
\cline { 2 - 8 } & Soja & Milho & Girassol & Arroz & Algodão & Oliva & Canola \\
\hline 20,0 & 59,0 & 67,6 & 58,3 & 73,8 & 67,7 & 79,7 & 73,1 \\
30,0 & 41,2 & 47,4 & 41,3 & 50,5 & 47,3 & 55,4 & 50,5 \\
40,0 & 29,5 & 32,3 & 29,1 & 34,3 & 33,4 & 37,8 & 35,6 \\
50,0 & 22,3 & 24,8 & 21,3 & 24,5 & 24,6 & 26,2 & 25,2 \\
60,0 & 16,7 & 18,5 & 16,4 & 19,2 & 18,0 & 21,4 & 19,1 \\
70,0 & 12,6 & 14,0 & 12,6 & 14,2 & 14,0 & 14,9 & 14,5 \\
\hline
\end{tabular}

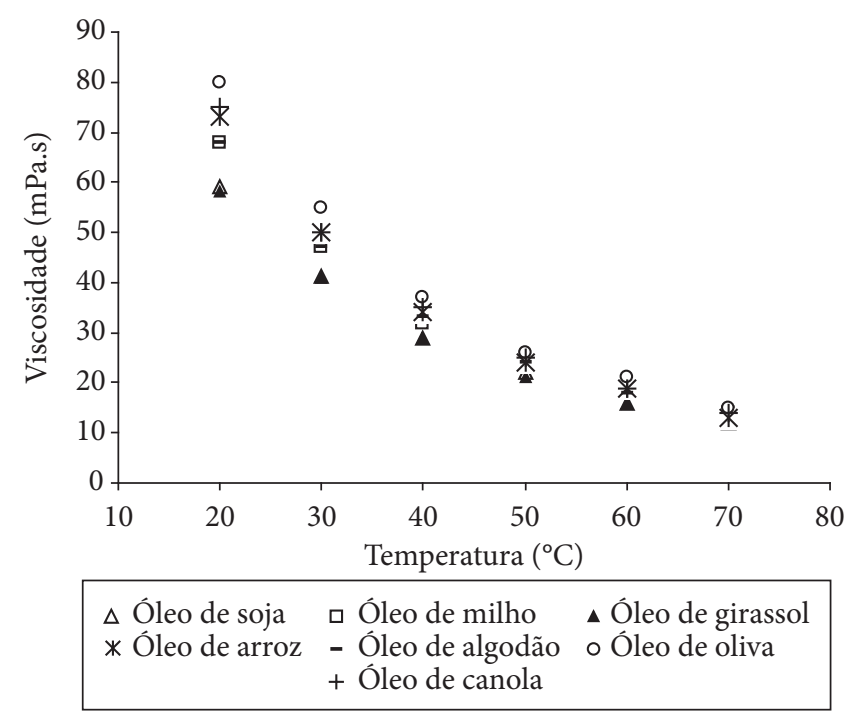

Figura 4. Valores de viscosidade para os diferentes óleos vegetais. 
$\ln \eta=A+\frac{B}{T}$

$\ln \eta=A+\frac{B}{C+T}$

em que:

$\eta=$ viscosidade dinâmica;

A, B e C = parâmetros ajustáveis; e

$\mathrm{T}=$ temperatura do sistema.

Para a regressão dos dois modelos (Equações 7 e 8), a seguinte função de mínimos quadrados foi minimizada (Equação 9), usando-se o solver do Excel $^{\oplus}$ :

$F O=\sum_{i=1}^{N O B S}\left(\eta_{i}^{\text {Calc }}-\eta_{i}^{E x p}\right)^{2}$

em que, $\eta i^{E x p}$ e $\eta i^{\text {Calc }}$ são os valores de viscosidade experimentais e calculados, respectivamente, e NOBS denota o número de observações experimentais utilizadas; tais parâmetros são apresentados na Tabela 4 .

A Figura 5 apresenta a comparação entre os valores experimentais da viscosidade para o óleo de soja e ambas as curvas dos modelos ajustados.

Uma vez que o modelo de Vogel mostrou-se mais adequado para representar a viscosidade em função da temperatura, este foi então empregado para representar os dados de viscosidade

Tabela 4. Parâmetros dos modelos de viscosidade para o óleo de soja.

\begin{tabular}{lcccc}
\hline \multicolumn{1}{c}{ Modelo } & A & B & C & DMA $(\%)^{*}$ \\
\hline Modelo de Guzman & 11,3005 & 33,8079 & - & 15,6 \\
Modelo de Vogel & 0,6814 & 464,94 & 83,9882 & 3,1 \\
\hline *Desvio médio absoluto percentual: & $\frac{100}{\text { NOBS }} \times \sum_{i}^{\text {NoBs }} \frac{\eta_{i}^{\text {Exp }}-\eta_{i}^{\text {Calc }} \mid}{\eta_{i}^{\text {Exp }}}$.
\end{tabular}

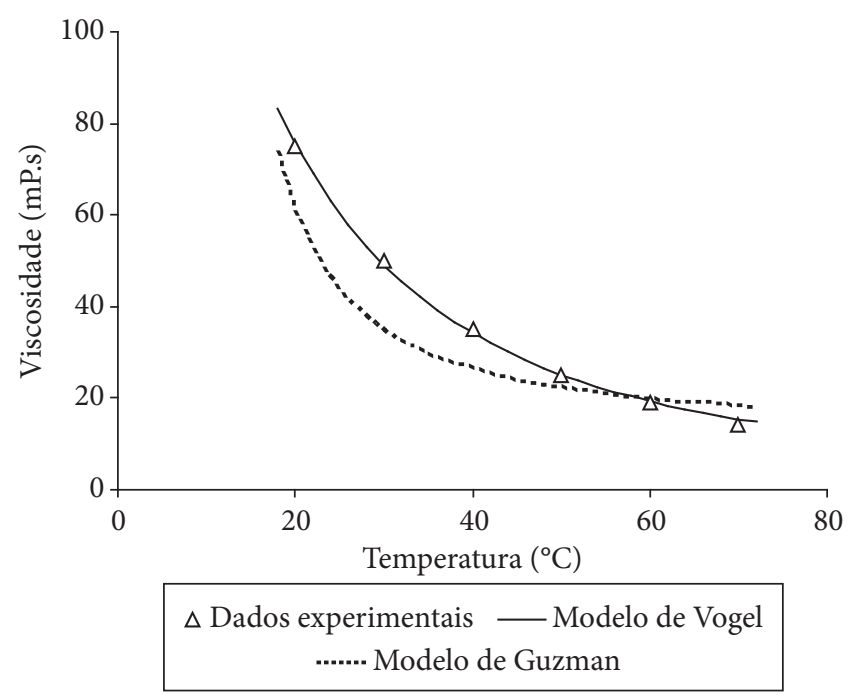

Figura 5. Valores de viscosidade para os diferentes óleos vegetais: experimental e modelos ajustados para o óleo de soja. dos demais óleos vegetais. A Tabela 5 apresenta os resultados da regressão para os demais óleos vegetais utilizando o modelo de Vogel.

\subsection{Medidas de condutividade e difusividade térmica}

Na Tabela 6 são apresentados os valores de condutividade (k) térmica medidos para os óleos vegetais investigados em diferentes temperaturas. A título ilustrativo, a Figura 6 fornece uma visualização dos valores desta propriedade em função da temperatura para os óleos de algodão e de soja. Para os valores de $\mathrm{k}$ foi utilizado um modelo linear para correlacionar os valores experimentais da condutividade térmica em função da temperatura. Na Tabela 7 são apresentados os valores dos coeficientes do modelo linear, bem como o coeficiente de correlação do modelo $\left(\mathrm{R}^{2}\right)$.

Para todos os óleos vegetais investigados neste trabalho os valores de difusividade térmica $(\alpha)$ medidos foram de $0,1( \pm 0,01) \mathrm{mm}^{2} \cdot \mathrm{s}^{-1}$. Para os valores de $\alpha$ a estratégia de correlação com um modelo linear não foi empregada dada a constância apresentada por esta propriedade em função da temperatura.

A partir da Figura 6 é possível observar que a condutividade térmica dos diferentes óleos vegetais apresenta, de uma maneira geral, comportamento praticamente linear em função da temperatura e que, no intervalo de temperatura investigado, verificou-se apenas uma ligeira redução dos valores desta propriedade em função da variável de medida. A difusividade térmica, por sua vez, apresentou fraca dependência com a temperatura, dentro do limite da precisão experimental de medida de tal propriedade.

De maneira geral, tanto a condutividade quanto a difusividade térmica não apresentaram diferenças significativas em relação aos diferentes óleos vegetais avaliados, indicando assim que as diferenças de composição química existentes entre os óleos vegetais investigados não influenciam significativamente os valores das propriedades térmicas mensuradas.

\section{Conclusões}

Neste trabalho foram avaliadas propriedades termofísicas de diferentes óleos vegetais refinados, em que foram medidas a viscosidade, condutividade e difusividade térmica em diferentes temperaturas. A partir dos resultados verificou-se que a

Tabela 5. Parâmetros do modelo de Vogel de viscosidade para os óleos vegetais.

\begin{tabular}{lcccc}
\hline \multirow{2}{*}{ Óleo } & \multicolumn{2}{c}{ Parâmetros do modelo de Vogel } & \multirow{2}{*}{ DMA $(\%)^{*}$} \\
\cline { 2 - 4 } & $\mathrm{A}$ & $\mathrm{B}$ & $\mathrm{C}$ & \\
\hline Milho & 0,6455 & 479,02 & 82,6303 & 3,5 \\
Girassol & 0,6226 & 478,74 & 85,2979 & 2,8 \\
Arroz & 0,6210 & 479,41 & 80,2884 & 6,1 \\
Algodão & 0,6606 & 479,11 & 83,1437 & 3,3 \\
Oliva & 0,6867 & 479,67 & 80,5015 & 6,4 \\
Canola & 0,6351 & 479,50 & 80,3320 & 3,0 \\
\hline *Desvio médio absoluto percentual: & $\frac{100}{N O B S} \times \sum_{i}^{\text {NoBs }} \frac{\eta_{i}^{\text {Exp }}-\eta_{i}^{\text {Calc }} \mid}{\eta_{i}^{\text {Exp }}}$ \\
\cline { 2 - 3 }
\end{tabular}


Tabela 6. Valores experimentais de condutividade térmica para os óleos vegetais em diferentes temperaturas.

\begin{tabular}{|c|c|}
\hline $\mathrm{T}\left({ }^{\circ} \mathrm{C}\right)$ & $\mathrm{k}(\mathrm{W} / \mathrm{m} . \mathrm{s}) \pm \sigma^{*}$ \\
\hline \multicolumn{2}{|l|}{ Óleo de algodão } \\
\hline 21,7 & $0,165 \pm 0,010$ \\
\hline 30,8 & $0,155 \pm 0,011$ \\
\hline 40,5 & $0,148 \pm 0,012$ \\
\hline 50,3 & $0,150 \pm 0,009$ \\
\hline 59,5 & $0,148 \pm 0,011$ \\
\hline 69,7 & $0,150 \pm 0,011$ \\
\hline \multicolumn{2}{|c|}{ Óleo de farelo de arroz } \\
\hline 21,4 & $0,160 \pm 0,015$ \\
\hline 31,3 & $0,155 \pm 0,011$ \\
\hline 40,5 & $0,155 \pm 0,010$ \\
\hline 50,5 & $0,158 \pm 0,008$ \\
\hline 60,1 & $0,145 \pm 0,012$ \\
\hline 69,5 & $0,143 \pm 0,013$ \\
\hline \multicolumn{2}{|l|}{ Óleo de girassol } \\
\hline 21,0 & $0,165 \pm 0,010$ \\
\hline 30,5 & $0,160 \pm 0,011$ \\
\hline 41,5 & $0,157 \pm 0,014$ \\
\hline 50,75 & $0,156 \pm 0,017$ \\
\hline 59,6 & $0,160 \pm 0,011$ \\
\hline 68,7 & $0,155 \pm 0,018$ \\
\hline \multicolumn{2}{|l|}{ Óleo de canola } \\
\hline 20,3 & $0,155 \pm 0,009$ \\
\hline 31,0 & $0,147 \pm 0,010$ \\
\hline 40,5 & $0,145 \pm 0,011$ \\
\hline 50,8 & $0,147 \pm 0,012$ \\
\hline 60,5 & $0,142 \pm 0,016$ \\
\hline 70,0 & $0,144 \pm 0,015$ \\
\hline \multicolumn{2}{|l|}{ Óleo de milho } \\
\hline 20,4 & $0,155 \pm 0,010$ \\
\hline 30,0 & $0,153 \pm 0,012$ \\
\hline 41,0 & $0,155 \pm 0,013$ \\
\hline 50,4 & $0,150 \pm 0,010$ \\
\hline 59,8 & $0,147 \pm 0,016$ \\
\hline 69,7 & $0,152 \pm 0,012$ \\
\hline \multicolumn{2}{|l|}{ Óleo de soja } \\
\hline 21,0 & $0,180 \pm 0,012$ \\
\hline 31,5 & $0,178 \pm 0,011$ \\
\hline 41,5 & $0,174 \pm 0,014$ \\
\hline 50,0 & $0,168 \pm 0,012$ \\
\hline 59,8 & $0,156 \pm 0,016$ \\
\hline 69,5 & $0,160 \pm 0,009$ \\
\hline \multicolumn{2}{|l|}{ Óleo de oliva } \\
\hline 21,8 & $0,166 \pm 0,013$ \\
\hline 31,5 & $0,158 \pm 0,012$ \\
\hline 40,5 & $0,159 \pm 0,015$ \\
\hline 50,1 & $0,149 \pm 0,012$ \\
\hline 59,5 & $0,155 \pm 0,016$ \\
\hline 68,9 & $0,150 \pm 0,010$ \\
\hline
\end{tabular}

${ }^{\star}$ Desvio padrão.

viscosidade dos óleos vegetais é significativamente influenciada pela temperatura, pois um aumento desta variável provoca uma diminuição sensível na viscosidade dos óleos vegetais. Com relação ao comportamento reológico, os diferentes óleos vegetais podem ser classificados como Newtonianos. No que se refere à densidade, observou-se que não há diferença significativa

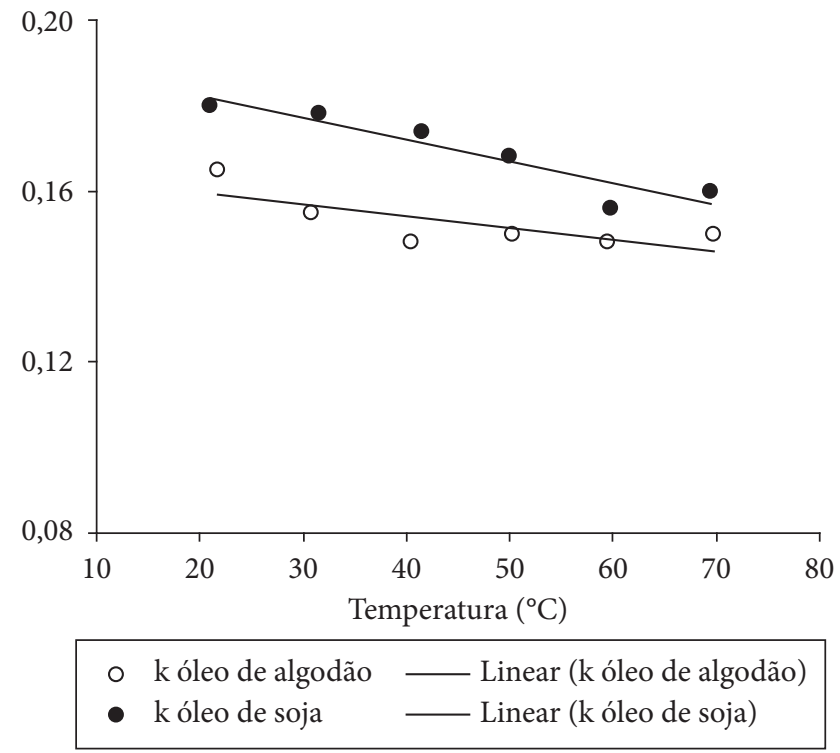

Figura 6. Condutividade térmica para os óleos de algodão e de soja em função da temperatura.

Tabela 7. Coeficientes do modelo linear ajustado para representação da condutividade térmica dos diferentes óleos vegetais.

\begin{tabular}{|c|c|c|c|}
\hline \multirow[t]{2}{*}{ Óleo } & \multicolumn{2}{|c|}{$\begin{array}{l}\text { Coeficientes do modelo linear } \\
\qquad(\mathrm{k}=\mathrm{a} \times \mathrm{T}+\mathrm{b})\end{array}$} & \multirow[t]{2}{*}{$\operatorname{DMA}(\%)^{\star}$} \\
\hline & $\mathrm{a} \times 10^{3}$ & $\mathrm{~b} \times 10^{2}$ & \\
\hline Algodão & $-0,3$ & 16,53 & 2,03 \\
\hline Arroz & $-0,3$ & 16,78 & 1,89 \\
\hline Girassol & $-0,2$ & 16,59 & 1,38 \\
\hline Canola & $-0,2$ & 15,56 & 1,56 \\
\hline Milho & $-0,1$ & 15,69 & 1,23 \\
\hline Soja & $-0,5$ & 19,25 & 1,51 \\
\hline Oliva & $-0,3$ & 16,98 & 1,73 \\
\hline
\end{tabular}

no valor desta propriedade em relação à matriz oleaginosa. Também, o mesmo fato foi verificado para a condutividade e difusividade térmica dos óleos vegetais nas diferentes temperaturas investigadas. Os resultados reportados neste trabalho podem ser de grande valia no projeto e operação de processos envolvendo óleos vegetais.

\section{Referências bibliográficas}

CONCEIÇÃO, M. M. et al. Rheological Behavior of Castor Oil Biodiesel. Energy \& Fuels, v. 19, n. 5, p. 2185-2188, 2005.

SANTOS, J. C. O.; SANTOS, I. M. G.; SOUZA, A. G. Effect of heating and cooling on rehological parameters of edible vegetable oils. Journal of Food Enginnering, v. 67, n. 4, p. 401-405, 2005.

ENCINAR, J. M. et al. Biodiesel Fuels from Vegetable Oils: Transesterification of Cynara cardunculus L. Oils with Ethanol. Energy \& Fuels, v. 16, n. 2, p. 443-450, 2002.

EITMAN, M. A.; GOODRUM, J. W. Rheology of the triglycerides tricaproin, tricaprylin, tricaprin and of diesel fuel, Transactions ASAE, v. 36, n. 2, p. 503-507, 1993. 
EITMAN, M. A.; GOODRUM, J. W. Density and viscosity of lowmolecular-weight triglycerides and their mixtures. Journal of American Oil Chemists Society, v. 71, n. 1, p. 1261-1265, 1994.

GOODRUM, J. W.; EITMAN, M. A. Physical properties of low molecular weight triglycerides for the development of biodiesel fuel models. Bioresource Technology, v. 56, n. 1, p. 55-60, 1996.

GELLER, D. P.; GOODRUM, J. W. Rheology of vegetable oil analogs and triglycerides. Journal of American Oil Chemists Society, v. 77, n. 2, p. 111-114, 1998.

VALERI, D.; MEIRELLES, A. J. A. Viscosities of fatty acids, triglycerides and their binary mixtures. Journal of American Oil Chemists Society, v. 74, n. 10, p. 1221-1226, 1997.

WU, J. et al. Thermal conductivity of some oxygenated fuels and additives in the saturated liquid phase. Journal of Chemical and Engineering Data, v. 50, n. 1, p. 102-104, 2005.
RABELO, J. et al. Viscosity prediction for fatty systems. Journal of American Oil Chemists Society, v. 77, n. 12, p. 1255-1262, 2000.

RESENDE, J. V.; SILVEIRA JR., V. Medidas da condutividade térmica de modelos de poupas de frutas no estado congelado. Ciência e Tecnologia de Alimentos, v. 22, n. 2, p. 177-183, 2002.

FONTANA, A. J. et al. Simultaneous Thermal Conductivity, Thermal Resistivity, and Thermal Diffusivity Measurement of Selected Foods and Soils. California, USA: ASAE (The Society for engineering in agricultural, food, and biological systems), 2001.

INCROPERA, F. P.; DE WITT, D. P. Introduction to Heat Transfer. 2 ed. New York, USA: John Wiley \& Sons, 1990.

WELTY, J. R.; WICKS, C. E.; WILSON, R. E. Fundamentals of Momentum, Heat and Mass Transfer. New York, USA: John Wiley \& Sons, 1984.

REID, R. C.; PRAUSNITZ, J. M.; POLING, B. E. The Properties of Gases \& Liquids. 4 ed. New York, USA: McGraw-Hill, 1988. 